\title{
Case selection and implementation of tubeless percutaneous nephrolithotomy
}

\author{
Yun-Hua Mao ${ }^{1 \#}$, Chun-Ping Huang ${ }^{2 \#}$, Teng-Cheng Li $^{1}$, Wen-Biao Li ${ }^{1}$, Fei Yang ${ }^{1}$, Bo-Long Liu ${ }^{1}$, \\ Yun Luo ${ }^{1}$, Xue-Lian Chen ${ }^{1}$, Hai-Lun Zhan ${ }^{1 \wedge}$ \\ ${ }^{1}$ Department of Urology, Lingnan Hospital, Third Affiliated Hospital of Sun Yat-sen University, Guangzhou, China; ${ }^{2}$ Department of Respiratory \\ Medicine, The Central Hospital of Panyu, Guangzhou, China \\ Contributions: (I) Conception and design: HL Zhan; (II) Administrative support: HL Zhan, Y Luo; (III) Provision of study materials or patients: YH \\ Mao, TC Li, WB Li, F Yang, BL Liu; (IV) Collection and assembly of data: CP Huang, XL Chen; (V) Data analysis and interpretation: YH Mao, CP \\ Huang; (VI) Manuscript writing: All authors; (VII) Final approval of manuscript: All authors. \\ \#These authors contributed equally to this work. \\ Correspondence to: Hai-Lun Zhan; Xue-Lian Chen. Department of Urology, Lingnan Hospital, Third Affiliated Hospital of Sun Yat-sen University, \\ Guangzhou 510630, China. Email: zhanhl@mail.sysu.edu.cn; chxuel@mail.sysu.edu.cn.
}

Background: The tubeless percutaneous nephrolithotomy (PCNL) was proposed to eliminate the side effects of the nephrostomy tube in recent years, such as pain, channel infection, postoperative bleeding, and longer hospital stay. But there is neither clinical guidelines nor consensus about tubeless PCNL in clinical practice. The study is aimed to how to implement the tubeless PCNL step by step, including case selection preoperatively, improving the technique of the surgeon, making the correct decisions at the end of the procedure, which had not been previously examined.

Methods: From January 2017 to March 2018, 364 consecutive patients requiring PCNL were comprehensively analyzed preoperatively and patients were selected for scheduled tubeless PCNL based on four aspects. The selected patients were divided into two groups according to whether the nephrostomy tube was finally placed. The mean operative time, intraoperative blood loss, stone clearance rate, visual pain score, postoperative hospitalization days and perioperative complications were all evaluated.

Results: Based on the preoperative evaluation, 42 patients were selected for tubeless PCNL, among which there were finally 37 cases of completed tubeless PCNL. Compared with patients undergoing conventional PCNL, there were not statistical differences in the mean operative time $(\mathrm{P}=0.207)$ or intraoperative blood loss $(\mathrm{P}=0.450)$ in the tubeless group. Stone clearance rate was $100 \%$ in both groups. The visual pain scores in the tubeless PCNL group were lower on operation day $(\mathrm{P}=0.029)$, first postoperative day $(\mathrm{P}<0.001)$ and the day of discharge $(\mathrm{P}=0.025)$. The postoperative hospitalization for the tubeless $\mathrm{PCNL}$ group was shorter than that of the control group $(\mathrm{P}<0.001)$. No significant difference in grade 1 complications was seen $(\mathrm{P}=0.424)$, and no grade 2 or higher complications were observed in either group.

Conclusions: Postoperative pain was significantly relieved and postoperative hospitalization was significantly shortened in the tubeless PCNL group. Tubeless PCNL is safe if patients are carefully selected using four criteria before operation, attention is paid to four key points and five confirmations are made during operation.

Keywords: Renal stones; tubeless percutaneous nephrolithotomy (PCNL); patient selection; intraoperative decision

Submitted Jun 02, 2021. Accepted for publication Jul 27, 2021.

doi: $10.21037 /$ tau-21-559

View this article at: https://dx.doi.org/10.21037/tau-21-559

^ ORCID: 0000-0001-9094-2250. 


\section{Introduction}

Minimally invasive percutaneous nephrolithotomy (PCNL) has been widely used in the treatment of renal and upper ureteral calculi (1). Nephrostomy and double-J tubes are routinely placed after PCNL surgery, but the nephrostomy tube is associated with adverse events such as pain, channel infection, postoperative bleeding, and longer hospital stay. In 1986, Dickinson et al. first proposed the concept of tubeless PCNL to eliminate the side effects of the nephrostomy tube (2). However, most clinicians believe its application should be limited to strictly selected patients (3). Currently, with improvements in PCNL and the emphasis on enhanced recovery after surgery, tubeless PCNL have been attracting more and more attention (4). However, to date there is neither clinical guidelines nor consensus about tubeless PCNL in clinical practice (5). Here, we report our experience with choosing patients for tubeless PCNL preoperatively and how to make the correct decisions to ensure postoperative safety during the operation. We present the following article in accordance with the STROBE reporting checklist (available at https://dx.doi. org/10.21037/tau-21-559).

\section{Methods}

\section{Case selection and patients' data}

This prospective study was approved by the institutional ethics committee of the Third Affiliated Hospital of Sun Yat-sen University and all patients signed the informed consent form for use of their related information. The study was conducted in accordance with the Declaration of Helsinki (as revised in 2013). From January 2017 to March 2018, a total of 364 consecutive cases requiring PCNL were analyzed preoperatively. Preoperative evaluation of all patients included disease history, physical examination, routine laboratory investigations, imaging examination including renal ultrasonography (US), plain radiography of kidneys-ureters-bladder (KUB), intravenous urography (IVU) and spiral CT urography (CTU). Urine cultures were obtained. Preoperatively, we identified several conditions that were not appropriate for tubeless PCNL: (I) ureteropelvic junction stenosis or ureteral stenosis on the operation side; (II) complex renal stones that could be difficult to remove at one time; (III) renal cortical thickness $<4 \mathrm{~mm}$; (IV) abnormal coagulation function; and (V) obvious preoperative infection. According to these comprehensive evaluation criteria, a total of 42 patients were scheduled to undergo tubeless PCNL.

\section{Surgical method}

Routine tracheal intubation with the patient under general anesthesia was performed and generally, the surgical procedure was as previously described (6). A $5 \mathrm{~F}$ ureteral catheter was placed retrogradely into the diseased renal pelvis using a cystoscope in the lithotomy position. The patient was then placed in the prone position and the target renal calyx was punctured using an $18-\mathrm{G}$ renal puncture needle under real-time ultrasound guidance. If urine/saline spilled when the needle core was withdrawn, the needle tip was in the correct position. A 0.035 -inch guidewire (COOK) was inserted into the needle sheath until it reached the renal pelvis. The needle sheath was withdrawn, and the tract was then dilated to $18 \mathrm{Fr}$ using fascial dilators (COOK, 6-18 Fr). A 'peel-away sheath' (COOK, 18 Fr) was inserted as the working tract. The ureteroscope (Olympus, $9.8 \mathrm{~F}$ ) was inserted into the working sheath to locate the calculi, which were fragmented using a holmium laser lithotripsy device. For staghorn calculi and multiple stones, if it proved difficult to completely remove the calculus with a single working tract, a second working tract was established under ultrasound guidance to facilitate removal of the stones and shorten the operation time.

\section{Intraoperative decisions}

During the operation, there were several points at which it had to be determined whether the nephrostomy tubes were finally placed or not. Patients were selected intra-operatively for tubeless PCNL based on our decision-making protocol (Figure 1). If the renal collecting system was damaged or the ureter, especially the ureteropelvic junction, was narrow, a nephrostomy tube was placed after the operation.

After the calculi were extracted, ultrasound was used to confirm clearance. If the stones were not removed completely, a nephrostomy tube was also placed.

Antegrade placement of a 6-F double-J catheter was performed after confirming that all calculi in the observable range had been removed through microscopic and ultrasound exploration. A security guidewire was placed to the renal pelvis and under the ureteroscopic guidance, the peel-away sheath was pulled back to the margin of the renal calyx, followed by reducing the washing pressure or ceasing the washing. The surgeon carefully confirmed there was no active bleeding while pulling the sheath back out of the 


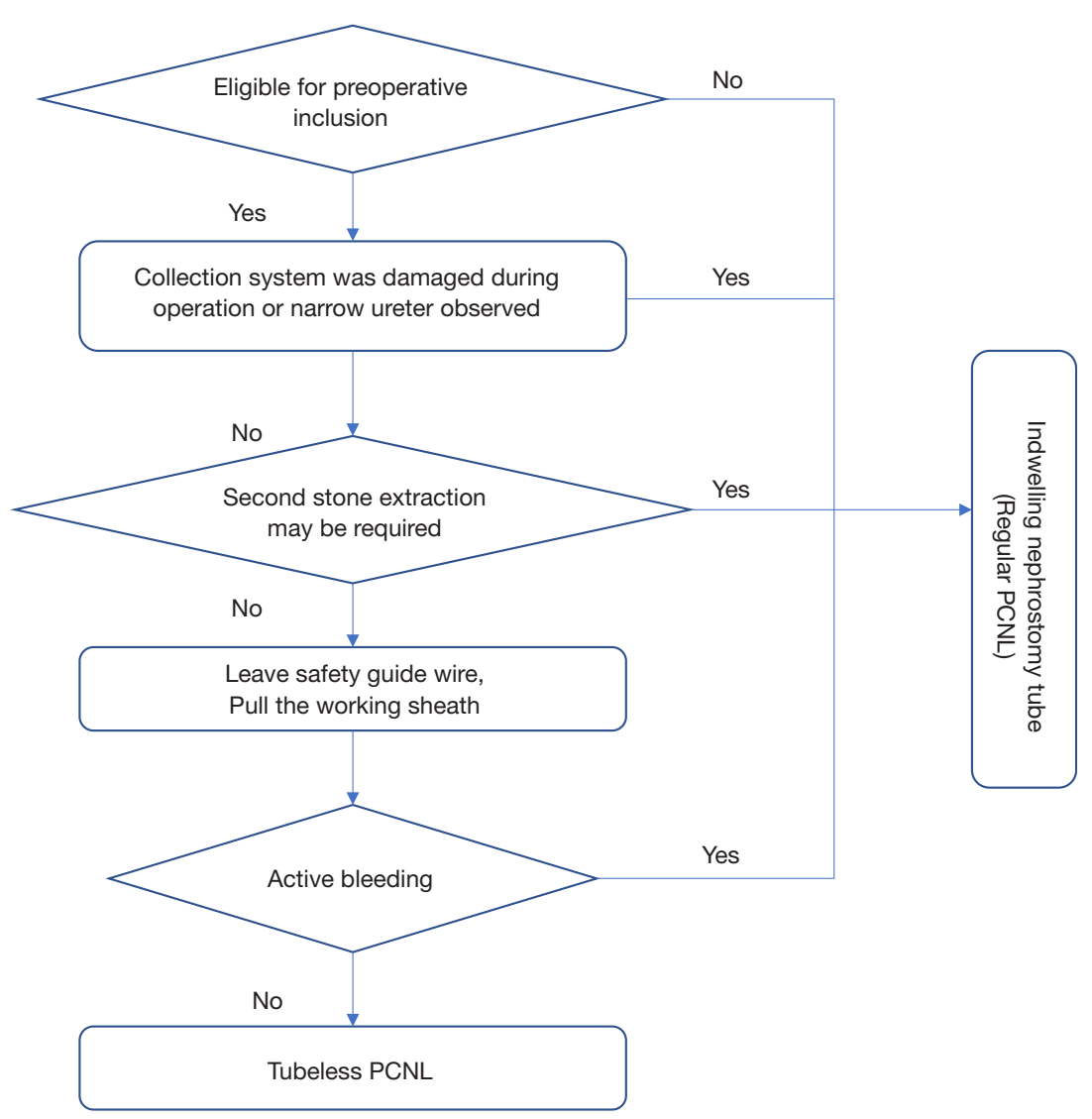

Figure 1 Intraoperative decision-making process. PCNL, percutaneous nephrolithotomy.

incision. Another 5-min observation of incision bleeding is important. If there was significant hemorrhage around the channel, especially arterial bleeding or massive venous bleeding, a nephrostomy tube was placed postoperatively. If there was no significant hemorrhage around the channel, the nephrostomy tube was not necessary and the security guidewire was removed and the operation was completed. The incision of the nephrostomy tract is not closed when tubeless nephrostomy is performed, to allow the drainage of fluid and avoid postoperative fever.

\section{Statistical analysis}

According to whether patients were selected intraoperatively for tubeless PCNL or not, the patients who met the preoperative inclusion conditions were divided into a tubeless PCNL group and regular PCNL group, and the following indexes were evaluated: (I) operation time; (II) intraoperative blood loss (mL); (III) stone clearance rate: KUB was routinely performed on postoperative day 2 and patients were considered stone-free when no stone $>4 \mathrm{~mm}$ was visualized on KUB; (IV) visual analog scale (VAS) of pain. Pain severity was evaluated by a trained nurse using the VAS every $8 \mathrm{~h}$. The preoperative and postoperative VAS scores were recorded. The degree of pain from mild to severe was divided into 10 grades, no pain $=0$ points, the most serious pain $=10$ points; $(\mathrm{V})$ postoperative hospitalization days; and (VI) perioperative complications: the modified Clavien grading system proposed in 2004 was adopted in this study (7).

SPSS 19.0 software was used for the statistical analysis. Fisher's exact test or the $\chi^{2}$ test, as appropriate, was used for statistical analysis of the frequency data. Measurement data are presented as mean \pm SD. The between-group differences in these mean values were analyzed by Student's $t$-test. $\mathrm{P}<0.05$ was regarded as statistically significant.

\section{Results}

According to the preoperative conditions for tubeless 
Table 1 Preoperative clinical data of patients scheduled to undergo tubeless percutaneous nephrolithotomy

\begin{tabular}{|c|c|}
\hline Variable & Number \\
\hline No. of patients & 42 \\
\hline Male: female & $31: 11$ \\
\hline Mean age (years) & $42.5 \pm 11.6$ \\
\hline \multicolumn{2}{|l|}{ Mean BMI } \\
\hline \multicolumn{2}{|l|}{ Right side/left side } \\
\hline Right & 19 \\
\hline Left & 23 \\
\hline \multicolumn{2}{|l|}{ Stones } \\
\hline Single upper ureteral calculus & 5 \\
\hline Single renal pelvis calculus & 7 \\
\hline $\begin{array}{l}\text { Single calyx calculus combined with } \\
\text { renal pelvis calculi }\end{array}$ & 16 \\
\hline $\begin{array}{l}\text { Two calyces calculi combined with renal } \\
\text { pelvis calculi }\end{array}$ & 8 \\
\hline Complete staghorn calculus & 6 \\
\hline \multicolumn{2}{|l|}{ Hydronephrosis } \\
\hline Mild & 5 \\
\hline Moderate & 26 \\
\hline Severe & 11 \\
\hline \multicolumn{2}{|l|}{ Surgical history } \\
\hline Open nephrolithotomy & 3 \\
\hline PCNL & 3 \\
\hline Extracorporeal shockwave lithotripsy & 2 \\
\hline None & 34 \\
\hline
\end{tabular}

PCNL, percutaneous nephrolithotomy; BMI, body mass index.

PCNL, 42 of 364 patients were eligible for inclusion in this study. Their clinical data are shown in Table 1.

Among them, 37 patients finally underwent tubeless PCNL, and 5 patients required placement of a nephrostomy tube. All operations were successful. The mean operative time $(\mathrm{P}=0.207)$ and intraoperative blood loss $(\mathrm{P}=0.450)$ of the two groups were not statistically significant. The stone clearance rate was $100 \%$ in both groups. Representative KUB of pre- and post-operation are shown in Figure $2 A, B$. Only two cases of grade 1 complications occurred in the tubeless PCNL group, and only one case in the PCNL group. No grade 2 or higher complications occurred in either group. Blood transfusion was not performed in either group.

The VAS pain score of the tubeless PCNL group was significantly less than that of the PCNL group, regardless of whether it was the operation day $(\mathrm{P}=0.029)$, postoperative day $1(\mathrm{P}<0.001)$ or discharge day $(\mathrm{P}=0.025)$. Furthermore, the mean hospital stay was significantly shorter in the tubeless PCNL group than in the PCNL group $(\mathrm{P}<0.001)$ (Table 2).

\section{Discussion}

In 1986, Dickinson et al. first proposed the concept of tubeless PCNL, aiming to reduce postoperative hemorrhage, channel infection, urinary extravasation, catheter pain and other discomfort and complications caused by an indwelling nephrostomy tube, and thus shorten the length of hospital stay (2). Tubeless PCNL includes complete tubeless and partial tubeless. The former has a high risk of stone residues, obstruction and urinary extravasation, which may cause severe renal colic $(8,9)$. Therefore, the indications should be strictly controlled and the technical requirements are relatively high. In recent years, more attention has been paid to the application of partial tubeless PCNL; that is, no indwelling nephrostomy tube, only a double-J tube or urinary tube, to reduce the risk of postoperative complications as much as possible by guaranteeing effective drainage (10).

Clinical studies have shown that partial tubeless PCNL has certain advantages (11-13): (I) the patient recovers quickly, including early postoperative eating and walking activities, which shortens the length of hospital stay; (II) without an indwelling nephrostomy tube, postoperative pain and discomfort can be significantly controlled and the dosage of analgesics is lower; and (III) without an obvious wound in tubeless PCNL, patients can recover quickly without obvious postoperative complications. By shortening the length of hospital stay and controlling complications, patients can get back to normal life and work earlier and reduce the economic burden at the same time.

As we all know, there are greater risks after the operation of tubeless PCNL. For example, there is no postoperative fistula compression, so postoperative bleeding in the working channel is more likely. Postoperative obstruction of drainage, caused by small stones or small blood clots, is more likely to cause fever or even urinary sepsis when a nephrostomy tube has not been placed (14). Tubeless PCNL is also prone to obstruction that can lead to backaches. So, 

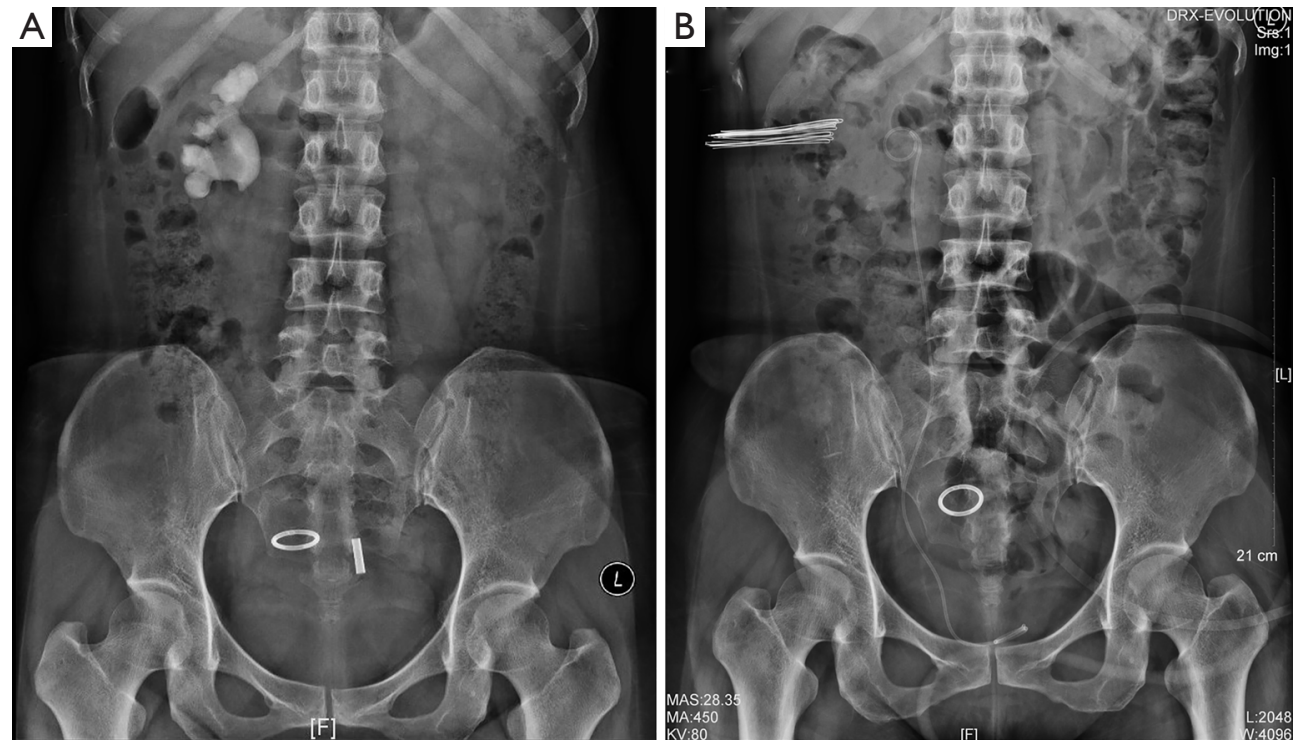

Figure 2 Representative KUB radiography pre- and post-operation. A 40-year-old female underwent single-tract tubeless PCNL. Preoperative KUB shows staghorn stone in the right kidney (A); postoperative KUB demonstrated no residual stones (B). KUB, kidneysureters-bladder; PCNL, percutaneous nephrolithotomy.

there were still some limitations of tubeless PCNL. The tubeless PCNL is not suit for all patients, because there are concerns that potentially fatal complications such as massive bleeding without a nephrostomy tube may occur. The tubeless PCNL must also be implemented by experienced surgeon. Therefore, it is an important clinical topic of how to safely implement tubeless PCNL. We believe that preoperative patient selection and intraoperative decision making are important and complementary in the implementation of tubeless PCNL.

\section{Preoperative patient selection and strict indications}

To date, there is no clear standard or guidelines on which patients can be treated with tubeless PCNL. According to our clinical experience, we suggest four types of patients who are indicated for PCNL, but are unsuitable for tubeless PCNL. (I) Renal cortical thickness $<4 \mathrm{~mm}$. Serious urinary extravasation is very common in these patients, because the thin renal cortex has difficulty shrinking and healing (15). (II) Abnormal coagulation function such as liver cirrhosis, longterm oral anticoagulant drugs, serious thrombocytopenia of varying etiology, and severe and longstanding diabetes leading to poor vascular elasticity (16). (III) Complex renal stones that could be difficult to remove at one time on the basis of preoperative prediction $(15,16)$. (IV) Serious urinary tract infection or obvious infectious stones, because insufficient drainage could escalate the infection or even contribute to urinary sepsis, which brings a high risk of death (17).

According to these strict indications, only 42 patients of 364 patients suitable for PCNL were indicated and scheduled for tubeless PCNL.

\section{Skilled and gentle operation to avoid complications}

Residual calculi and severe hemorrhage are the two major challenges to implementing tubeless PCNL. Avoiding intraoperative bleeding depends greatly on the experience and skill of the surgeon (18). Therefore, we recommend that tubeless PCNL should only be carried out by experienced surgeons.

We believe that attention should be paid to four key points during the operation, to avoid complications. (I) Accurate puncture: a good working channel is very helpful to remove the most stones and have the fewest complications $(19,20)$. (II) The channel should be gently expanded, and the direction and depth of channel should be correct rather than deep. (III) Avoid excessive swinging of the ureteroscope during the operation, which can result in severe hemorrhage by compressing the surrounding renal parenchyma or tearing the calyx neck by the working 
Table 2 Operative and postoperative outcomes of patients who underwent PCNL

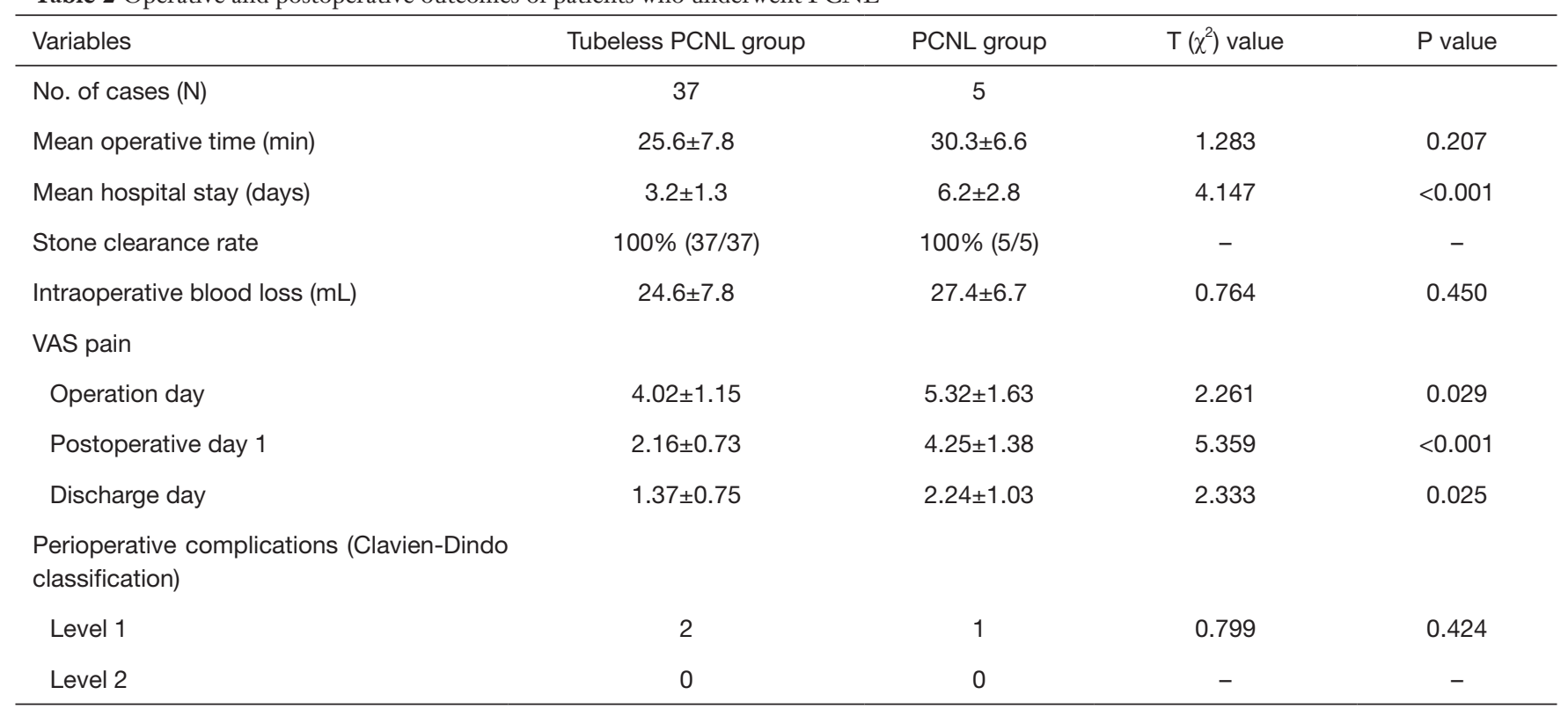

PCNL, percutaneous nephrolithotomy; VAS, visual analog scale.

sheath. It is very important to maintain a clear field of vision during the operation, which will reduce the incidence of residual calculi. (IV) The surgeon should be familiar with the calyces where the calculi are located and the calyces where the ureteroscope is located. This is helpful for finding residual calculi.

It is not easy to perform a perfect PCNL. Preoperative patient selection and strict indications is the premise. The main points of operation are accurate intraoperative puncture to establish the good working channel, and gentle operation during the operation to avoid complications. No complication is the premise for the tubeless PCNL at the end of procedure.

\section{Correct intraoperative decisions to ensure postoperative safety}

PCNL is complicated and can always change during the operation. Patients who were scheduled to undergo tubeless PCNL preoperatively will not always have tubeless PCNL implemented in the end. In order to ensure postoperative safety, a series of intraoperative decisions must be made. Based on our experience, we summarize the following five points that should be confirmed during operation. (I) Confirm the puncture of the calyx dome and establish a working channel along the central axis of the calyx, because the risk of postoperative bleeding in such a working channel is the lowest (21). (II) Confirm all the stones are removed without the need for a second-look for residual calculi (22). Before the end of the operation, routinely use ultrasound or X-ray to reevaluate for residual stones. (III) Confirm the absence of perforation in the collecting system. In the past, a nephrostomy tube would be placed in patients with a perforation in the collecting system or after multichannel PCNL to avoid urinary obstruction or even severe infection caused by poor drainage $(23,24)$. However, recent reports suggest that tubeless PCNL could be implemented after a minor perforation in the collecting system (25). It should be noted that two patients in our tubeless PCNL group had staghorn stones, and two working channels were used. The stone was confirmed to be completely removed and a nephrostomy tube was not placed. There was no obvious bleeding, urinary extravasation or other postoperative complications. Therefore, we believe that the tubeless PCNL can be also used in patients undergoing multichannel PCNL. (IV) Confirm that there is no ureteral stenosis, especially ureteropelvic junction obstruction (UPJO) to avoid postoperative obstruction. (V) After placement of the double-J tube, confirm there is no excessive bleeding around the working channel. The security guidewire should be placed to the renal pelvis and under ureteroscopic guidance, the peel-away sheath is pulled back to the margin of the renal calyx, followed by reducing the washing pressure or ceasing the washing. A 5 -min observation is strongly 
recommended to rule out significant hemorrhage around the channel, especially arterial bleeding or massive venous bleeding. Finally, the security guidewire is removed and the operation is completed. In brief, if all five points are met, the tubeless PCNL can be implemented and postoperative safety is guaranteed to the most extent.

In summary, tubeless PCNL has several advantages and is relatively safe, but it may have great risks if the patients are not carefully selected. Based on our experience, we propose that appropriate cases should be evaluated in four aspects preoperatively, attention should be paid to 4 key points during surgery, and 5 confirmations should be achieved. However, the selection criteria for tubeless PCNL still need to be further verified in prospective randomized controlled clinical trials.

\section{Acknowledgments}

Funding: This work is financially supported by Natural Science of Guangdong Province (S2013040014333).

\section{Footnote}

Reporting Checklist: The authors have completed the STROBE reporting checklist. Available at https://dx.doi. org/10.21037/tau-21-559

Data Sharing Statement: Available at https://dx.doi. org/10.21037/tau-21-559

Conflicts of Interest: All authors have completed the ICMJE uniform disclosure form (available at https://dx.doi. org/10.21037/tau-21-559). The authors have no conflicts of interest to declare.

Ethical Statement: The authors are accountable for all aspects of the work in ensuring that questions related to the accuracy or integrity of any part of the work are appropriately investigated and resolved. The study was conducted in accordance with the Declaration of Helsinki (as revised in 2013). This prospective study was approved by the institutional ethics committee of the Third Affiliated Hospital of Sun Yat-sen University (No. [2020]02-108-01X) and all patients signed the informed consent form for use of their related information.

Open Access Statement: This is an Open Access article distributed in accordance with the Creative Commons
Attribution-NonCommercial-NoDerivs 4.0 International License (CC BY-NC-ND 4.0), which permits the noncommercial replication and distribution of the article with the strict proviso that no changes or edits are made and the original work is properly cited (including links to both the formal publication through the relevant DOI and the license). See: https://creativecommons.org/licenses/by-nc-nd/4.0/.

\section{References}

1. Ahmad AA, Alhunaidi O, Aziz M, et al. Current trends in percutaneous nephrolithotomy: an internet-based survey. Ther Adv Urol 2017;9:219-26.

2. Dickinson IK, Fletcher MS, Bailey MJ, et al. Combination of percutaneous surgery and extracorporeal shockwave lithotripsy for the treatment of large renal calculi. Br J Urol 1986;58:581-4.

3. Wang J, Bai Y, Yin S, et al. Risk factors for deterioration of renal function after percutaneous nephrolithotomy in solitary kidney patients with staghorn calculi. Transl Androl Urol 2020;9:2022-30.

4. Zilberman DE, Lipkin ME, de la Rosette JJ, et al. Tubeless percutaneous nephrolithotomy--the new standard of care? J Urol 2010;184:1261-6.

5. Ghani KR, Andonian S, Bultitude M, et al. Percutaneous Nephrolithotomy: Update, Trends, and Future Directions. Eur Urol 2016;70:382-96.

6. Zhan HL, Li ZC, Zhou XF, et al. Supine lithotomy versus prone position in minimally invasive percutaneous nephrolithotomy for upper urinary tract calculi. Urol Int 2013;91:320-5.

7. Dindo D, Demartines N, Clavien PA. Classification of surgical complications: a new proposal with evaluation in a cohort of 6336 patients and results of a survey. Ann Surg 2004;240:205-13.

8. Istanbulluoglu MO, Ozturk B, Gonen M, et al. Effectiveness of totally tubeless percutaneous nephrolithotomy in selected patients: a prospective randomized study. Int Urol Nephrol 2009;41:541-5.

9. Moosanejad N, Firouzian A, Hashemi SA, et al. Comparison of totally tubeless percutaneous nephrolithotomy and standard percutaneous nephrolithotomy for kidney stones: a randomized, clinical trial. Braz J Med Biol Res 2016;49:e4878.

10. Marchant F, Recabal P, Fernandez MI, et al. Postoperative morbidity of tubeless versus conventional percutaneous nephrolithotomy: a prospective comparative study. Urol Res 2011;39:477-81. 
11. Shen P, Liu Y, Wang J. Nephrostomy tube-free versus nephrostomy tube for renal drainage after percutaneous nephrolithotomy: a systematic review and meta-analysis. Urol Int 2012;88:298-306.

12. Chen ZJ, Yan YJ, Zhou JJ. Comparison of tubeless percutaneous nephrolithotomy and standard percutaneous nephrolithotomy for kidney stones: A meta-analysis of randomized trials. Asian J Surg 2020;43:60-8.

13. Xun $\mathrm{Y}$, Wang Q, Hu H, et al. Tubeless versus standard percutaneous nephrolithotomy: an update meta-analysis. BMC Urol 2017;17:102.

14. Jou YC, Lu CL, Chen FH, et al. Contributing factors for fever after tubeless percutaneous nephrolithotomy. Urology 2015;85:527-30.

15. Ansari H, Tomar V, Yadav SS, et al. Study of predictive factors affecting the prolonged urinary leakage after percutaneous nephrolithotomy. Urol Ann 2016;8:60-5.

16. Akman T, Binbay M, Sari E, et al. Factors affecting bleeding during percutaneous nephrolithotomy: single surgeon experience. J Endourol 2011;25:327-33.

17. Shoshany O, Margel D, Finz C, et al. Percutaneous nephrolithotomy for infection stones: what is the risk for postoperative sepsis? A retrospective cohort study. Urolithiasis 2015;43:237-42.

18. Yu W, Rao T, Li X, et al. The learning curve for access creation in solo ultrasonography-guided percutaneous nephrolithotomy and the associated skills. Int Urol

Cite this article as: Mao YH, Huang CP, Li TC, Li WB, Yang F, Liu BL, Luo Y, Chen XL, Zhan HL. Case selection and implementation of tubeless percutaneous nephrolithotomy. Transl Androl Urol 2021;10(8):3415-3422. doi: $10.21037 /$ tau-21-559
Nephrol 2017;49:419-24.

19. Ng FC, Yam WL, Lim TYB, et al. Ultrasoundguided percutaneous nephrolithotomy: Advantages and limitations. Investig Clin Urol 2017;58:346-52.

20. Carrion DM, Cansino JR, Quintana LM, et al. Prone percutaneous nephrolithotomy: its advantages and our technique for puncture. Transl Androl Urol 2018;7:950-9.

21. Rodrigues PL, Rodrigues NF, Fonseca J, et al. Kidney targeting and puncturing during percutaneous nephrolithotomy: recent advances and future perspectives. J Endourol 2013;27:826-34.

22. Kim SC, Tinmouth WW, Kuo RL, et al. Using and choosing a nephrostomy tube after percutaneous nephrolithotomy for large or complex stone disease: a treatment strategy. J Endourol 2005;19:348-52.

23. Cho HJ, Lee JY, Kim SW, et al. Percutaneous nephrolithotomy for complex renal calculi: is multi-tract approach ok? Can J Urol 2012;19:6360-5.

24. Agrawal MS, Agrawal M. Tubeless percutaneous nephrolithotomy. Indian J Urol 2010;26:16-24.

25. Aghamir SMK, Salavati A, Hamidi M, et al. Primary Report of Totally Tubeless Percutaneous Nephrolithotomy Despite Pelvi-calyceal Perforations. Urol J 2017;14:4020-3.

(English Language Editor: K. Brown) 